\title{
DISCOVERY OF THREE DISTANT, COLD BROWN DWARFS IN THE WFC3 INFRARED SPECTROSCOPIC PARALLELS SURVEY
}

\author{
D. Masters ${ }^{1,2}$, P. McCarthy ${ }^{2}$, A. J. Burgasser ${ }^{3}$, N. P. Hathi ${ }^{2}$, M. Malkan $^{4}$, N. R. Ross ${ }^{4}$, B. Siana $^{1}$, C. Scarlata $^{5}$,

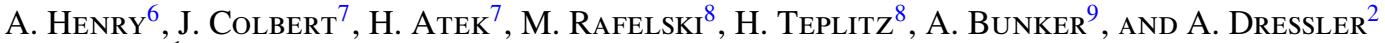 \\ ${ }^{1}$ Department of Physics and Astronomy, University of California, Riverside, CA 92521, USA \\ ${ }^{2}$ Observatories of the Carnegie Institution of Washington, Pasadena, CA 91101, USA \\ ${ }^{3}$ Center for Astrophysics and Space Science, University of California, San Diego, La Jolla, CA 92093, USA \\ ${ }^{4}$ Department of Physics and Astronomy, UCLA, Los Angeles, CA 90095, USA \\ ${ }^{5}$ Astronomy Department, University of Minnesota, Minneapolis, MN 55455, USA \\ ${ }^{6}$ Department of Physics, University of California, Santa Barbara, CA 93106, USA \\ ${ }^{7}$ Spitzer Science Center, Caltech, Pasadena, CA 91125 , USA \\ ${ }^{8}$ Infrared Processing and Analysis Center, Caltech, Pasadena, CA 91125, USA \\ ${ }^{9}$ Department of Physics, University of Oxford, Oxford, UK \\ Received 2012 April 10; accepted 2012 April 26; published 2012 May 25
}

\begin{abstract}
We present the discovery of three late-type ( $\geqslant \mathrm{T} 4.5)$ brown dwarfs, including a probable Y dwarf, in the WFC3 Infrared Spectroscopic Parallels (WISP) survey. We use the G141 grism spectra to determine the spectral types of the dwarfs and derive distance estimates based on a comparison with nearby $\mathrm{T}$ dwarfs with known parallaxes. These are the most distant spectroscopically confirmed $\mathrm{T} / \mathrm{Y}$ dwarfs, with the farthest at an estimated distance of $\sim 400 \mathrm{pc}$. We compare the number of cold dwarfs found in the WISP survey with simulations of the brown dwarf mass function. The number found is generally consistent with an initial stellar mass function $d N / d M \propto M^{-\alpha}$ with $\alpha=0.0-0.5$, although the identification of a Y dwarf is somewhat surprising and may be indicative of either a flatter absolute magnitude/spectral-type relation than previously reported or an upturn in the number of very-late-type brown dwarfs in the observed volume.
\end{abstract}

Key words: brown dwarfs - Galaxy: abundances - infrared: stars - stars: luminosity function, mass function

\section{INTRODUCTION}

Understanding the nature and demographics of lowtemperature brown dwarfs, which bridge the gap between dwarf stars and massive planets, is an important goal of modern astrophysics. Since the discovery of the first methane dwarf G1229B in 1995 (Nakajima et al. 1995; Oppenheimer et al. 1995), the number of known ultracool dwarfs of type $\mathrm{T}$ and now $\mathrm{Y}$ (Cushing et al. 2011) has continued to grow, primarily due to deep near-infrared surveys such as Two Micron All Sky Survey (Kirkpatrick et al. 1999; Burgasser et al. 2002), Sloan Digital Sky Survey (Strauss et al. 1999; Chiu et al. 2006), UKIDSS (Lodieu et al. 2007; Chiu et al. 2008; Burningham et al. 2010), CFBDS (Delorme et al. 2008), and WISE (Wright et al. 2010; Kirkpatrick et al. 2011). Additional dwarfs have been identified in proper-motion surveys (e.g., Kirkpatrick et al. 2010; Lucas et al. 2010; Scholz et al. 2011; Liu et al. 2011). These surveys have been effective at finding brown dwarfs in the solar neighborhood; however, due to their faintness (a T8 dwarf has $M_{A B} \sim 17$ in the $H$ band), probing the larger-scale galactic distribution of cold dwarfs has not been possible.

Deep surveys in the near-infrared using the Wide Field Camera 3 (WFC3) on the Hubble Space Telescope (HST) enable the discovery of cold dwarfs at much larger distances. WFC3 parallel imaging surveys have identified cold dwarfs based on broadband colors (e.g., Ryan et al. 2011), but the lack of spectroscopy means that the spectral types are uncertain. WFC3 near-infrared slitless grism spectroscopy, in contrast, permits unambiguous identification and classification of $\mathrm{T}$ dwarfs, whose spectra show pronounced atmospheric $\mathrm{CH}_{4}$ and $\mathrm{H}_{2} \mathrm{O}$ absorption bands in the near-infrared (Geballe et al. 2002; Burgasser et al. 2002). Moreover, the WFC3 spectra reach depths that are unachievable with ground-based surveys.
Here we present three of the most distant cold brown dwarfs known, discovered in the WFC3 Infrared Spectroscopic Parallels (WISP) survey. These discoveries illustrate that, by enabling spectroscopic identification of ultracool dwarfs at large distances, $H S T /$ WFC3 grism spectroscopy can begin to probe the galactic spatial distribution of the ultracool dwarf population and search for substellar members of the halo population (Burgasser et al. 2003).

\section{WISP SURVEY OVERVIEW}

The WISP survey (Atek et al. 2010) has obtained slitless spectra over $0.8-1.7 \mu \mathrm{m}$ for more than $700 \mathrm{arcmin}^{2}$ of sky using the two infrared grisms installed on the IR channel of WFC3. While the primary goal of WISP is to measure the star formation rate over $0.5<z<2.5$, the wide area and variety of galactic latitudes covered by WISP allow for the discovery of late-type dwarfs out to $\gtrsim 1 \mathrm{kpc}$.

WISP is a "pure parallel" program, meaning that the pointings are determined by other observing programs using either the Cosmic Origins Spectrograph or the Space Telescope Imaging Spectrograph. The WFC3 observations are taken in a parallel field determined by the offset of the instruments and the roll angle of the telescope. WISP is divided into a deep, narrow survey for parallel targets with more than four orbits of visibility and a shallow, wide survey for parallel targets with one to three orbits of visibility. Parallel observing targets are selected at galactic latitudes $|b|>20^{\circ}$ with a preference for longer visibility times. Typical integration times for a four-to five-orbit target are $\sim 5000 \mathrm{~s}$ in G102 and $2000 \mathrm{~s}$ in G141. The shallower WISP fields are restricted to the G141 grism, with a typical integration time for a two-orbit target of $\sim 4000 \mathrm{~s}$. Because the wide-shallow survey actually achieves deeper G141 integrations 

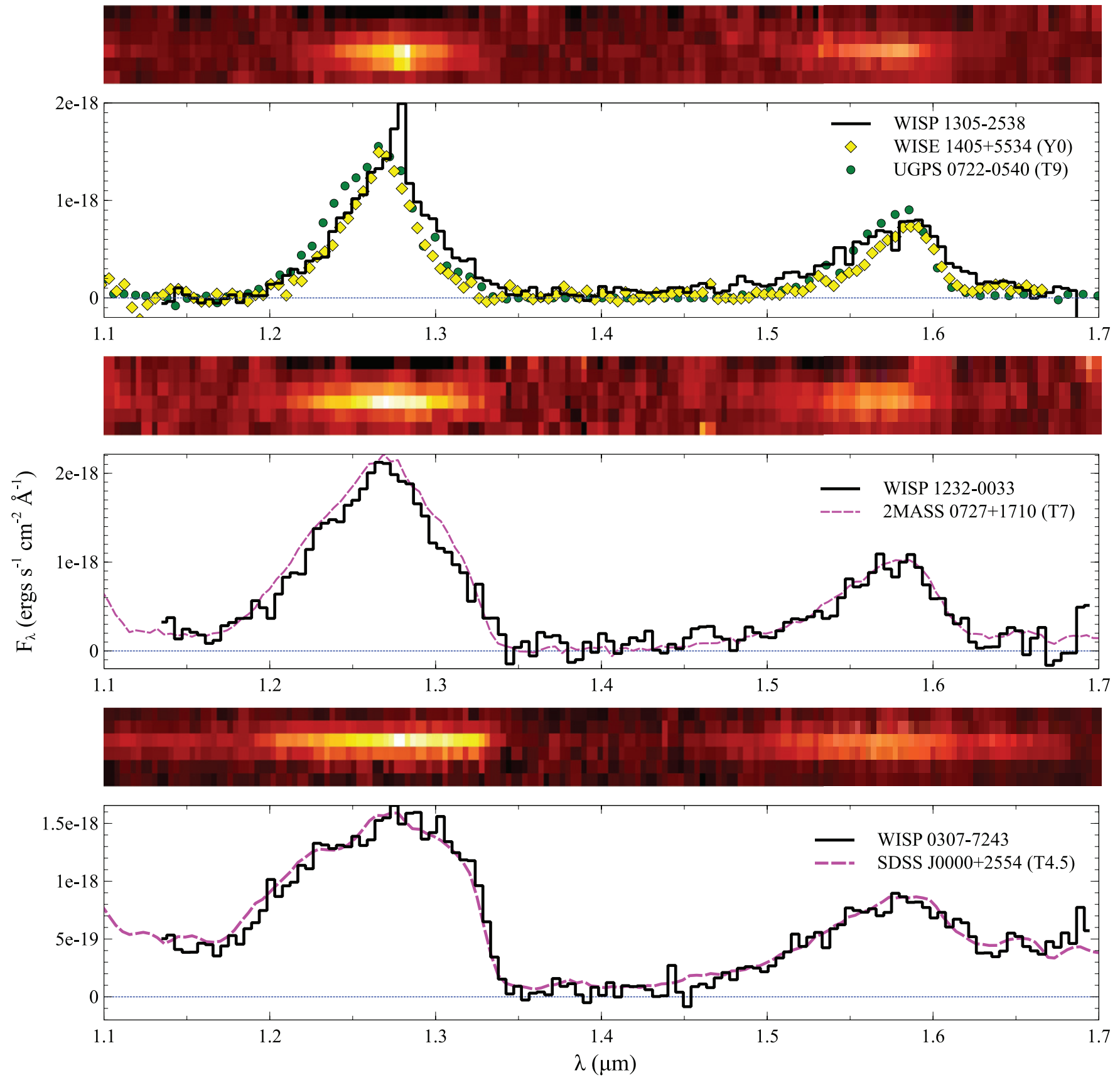

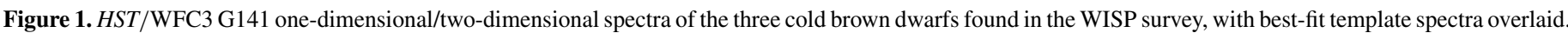

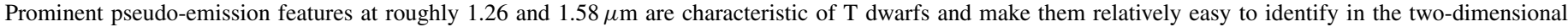

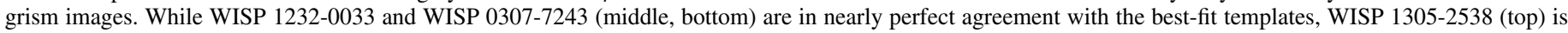

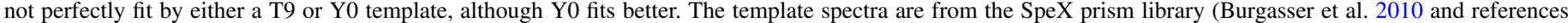
therein).

and covers a larger area than our deep-narrow survey, faint dwarfs are more likely to be found in the wide-shallow survey.

\section{ULTRACOOL DWARFS DISCOVERED IN THE WISP SURVEY}

Brown dwarfs of type $\gtrsim \mathrm{T} 2$ are easily recognizable in $H$-band spectroscopy due to the presence of two prominent, broad pseudo-emission features at roughly 1.26 and $1.58 \mu \mathrm{m}$ interspersed with deep absorption bands of water and methane in their atmospheres. The 155 G141 grism exposures (each $\left.123^{\prime \prime} \times 136^{\prime \prime}\right)$ currently obtained by the ongoing WISP survey were systematically scanned by at least two observers to search for these objects, resulting in the identification of the three sources shown in Figure 1. We note that another faint $\mathrm{T}$ dwarf was recently reported by the 3D-HST survey (Brammer et al. 2012), similarly found with deep WFC3 grism data. The properties of the three $\mathrm{T}$ dwarfs found here are summarized in Table 1.

\section{SPECTRAL CLASSIFICATION}

We determined the spectral types of the objects by comparing the WFC3 spectra to $69 \mathrm{~T}$ dwarf spectra from the SpeX Prism Spectral Libraries (Burgasser et al. 2006) and two additional Y dwarf spectra from Cushing et al. (2011), which were also 
Table 1

Cold Brown Dwarfs Discovered in the WISP Survey

\begin{tabular}{|c|c|c|c|c|c|c|c|}
\hline Name & $\begin{array}{l}\text { R.A. (J2000) } \\
\qquad \text { (h m s) }\end{array}$ & $\begin{array}{c}\text { Decl. (J2000) } \\
\left({ }^{\circ}{ }^{\prime \prime \prime}\right)\end{array}$ & $\begin{array}{l}\text { WFC3 Magnitude } \\
\text { (AB) }\end{array}$ & $\begin{array}{l}\text { MKO Magnitude } \\
\text { (AB) }\end{array}$ & Spectral-type & $\begin{array}{l}\text { Distance } \\
\quad(\mathrm{pc})\end{array}$ & $\begin{array}{c}\text { Galactic Latitude } \\
\text { (deg) }\end{array}$ \\
\hline WISP $1305-2538$ & 130525.51 & -253828.8 & $23.01 \pm 0.04^{\mathrm{a}}$ & $22.33 \pm 0.04^{b}$ & T9.5+ & $\sim 40-60^{\mathrm{c}}$ & +37.1 \\
\hline WISP 1232-0033 & 123242.42 & -003306.7 & $23.34 \pm 0.05^{\mathrm{a}}$ & $22.65 \pm 0.06^{\mathrm{b}}$ & $\mathrm{T} 7 \pm 0.6$ & $270 \pm 60$ & +63.1 \\
\hline WISP 0307-7243 & 030741.12 & -724357.5 & $22.67 \pm 0.02^{\mathrm{d}}$ & $22.36 \pm 0.02^{\mathrm{e}}$ & $\mathrm{T} 4.5 \pm 0.4$ & $400 \pm 60$ & -40.9 \\
\hline
\end{tabular}

Notes.

${ }^{\mathrm{a}} \mathrm{F} 140 \mathrm{~W}$.

b $J$ band.

$\mathrm{c}$ The interval arises from classification uncertainty and the current lack of quality parallaxes for Y dwarfs.

${ }^{\mathrm{d}}$ F160W.

e $H$ band.

obtained with the HST/WFC3 G141 grism using the same reduction software as the WISP survey spectra presented here. The WISP spectra and brown dwarf template spectra were compared using a $\chi^{2}$ statistic. The classifications and associated uncertainties were then found by computing the weighted mean and deviation from the mean with a weighting factor of $e^{\left(\min \left(\chi^{2}\right)-\chi^{2}\right)^{2}}$. A cutoff weight of $>0.1$ was used so that the results are not skewed by a large number of poor fits to early-type $\mathrm{T}$ dwarfs (a sampling problem that arises because the template sample contains disproportionately more early-type T dwarfs).

WISP 1232-0033 and WISP 0307-7243 are found to be type $\mathrm{T} 7 \pm 0.6$ and T4.5 \pm 0.4 , respectively. WISP $1305-2538$ is found to be of type T9.5+, and is probably a Y dwarf. The spectrum of the Y0 dwarf WISE 1405+5534 is shown overlaid in Figure 1, in addition to the T9 dwarf UGPS 0722-0540. While neither template is perfect, the $\mathrm{Y} 0$ spectrum is a better fit.

\section{DISTANCE DETERMINATION}

With accurate spectral types derived from the WFC3 spectra, the distances to the dwarfs can be found by comparing their measured broadband magnitudes with those of nearby $\mathrm{T}$ dwarfs with known parallaxes. For this comparison, we converted the WFC3 F140W/F160W photometry for the WISP dwarfs into the MKO $J$ or $H$ band with color corrections computed using the best-fitting $\mathrm{T}$ dwarf template spectra, applying the same weightings as for the spectral-type determination. Given the inferred MKO $\mathrm{J} / \mathrm{H}$ magnitudes, the distances were found using the absolute magnitude/spectral-type relations of Dupuy \& Liu (2012).

WISP 1232-0033 (T7) is found to be at $270 \pm 60 \mathrm{pc}$ and WISP $0307-7243$ (T4.5) at $400 \pm 60 \mathrm{pc}$. These are the most distant $\mathrm{T}$ dwarfs currently known, and have vertical scale heights of approximately 240 and 260 pc, respectively. WISP 1305-2538 is more difficult, as only one Y dwarf has reported a preliminary (and highly uncertain) parallax (WISE J154151.66-225025.2, $\pi=0.35 \pm 0$.'11; Kirkpatrick et al. 2011) and the classification scheme for these objects remains in progress (Cushing et al. 2011). We used the known parallaxes of the T9 UGPS 07220540 (0.'246 \pm 0.'0033; Lucas et al. 2010) and the possible Y dwarf (lacking spectroscopy) CFBDSIR J145829+101343B $(0$. 0433 \pm 0.'0045; Liu et al. 2011) to infer a distance of 40-60 pc for this source, albeit with considerable uncertainty because of the classification.

\section{SPACE DENSITY}

The dwarfs discovered in the WISP survey provide the first opportunity to examine the mass function and spatial distribution of these late-type dwarfs in a deep field (see Pirzkal et al. 2009 for an equivalent study of late M and L dwarfs). To assess what constraints our small sample makes on these statistics, we computed the expected surface densities of $\mathrm{T}$ dwarfs as a function of spectral type in the WISP survey fields as follows.

1. Volume densities for the cold dwarf subtypes are computed using the methods outlined in Burgasser (2004, 2007). We assume a power-law stellar mass function $d N / d M \sim$ $M^{-\alpha}$ with $\alpha$ varying from -1 to 1 , normalized to agree with measured field values in the $0.09-0.10 \mathrm{M}_{\odot}$ range (0.0055 $\mathrm{pc}^{-3}$; Reid et al. 1999; Chabrier 2001). We then assumed an age distribution consistent with a constant star formation rate over $10 \mathrm{Gyr}$, and computed the luminosity distribution for the dwarfs using the evolutionary models of Burrows et al. (1997). Finally, we use empirical bolometric correction/spectral-type (Liu et al. 2010) and absolute magnitude/spectral-type (Dupuy \& Liu 2012) relations to convert the luminosity function to a spectral-type surface density distribution.

2. The expected surface density of dwarfs per WISP $123^{\prime \prime} \times$ $136^{\prime \prime}$ pointing is then found by computing the limiting magnitude, and thus the maximum distance, for which each subtype would be spectroscopically detected ${ }^{10}$ in a given field. The online WFC3 exposure time calculator is used for this analysis. Corrections to the derived limiting magnitudes are applied to account for Malmquist bias. We assumed as a first-order approximation that $10 \%$ of each pointing is lost due to overlap, edge effects, etc., as estimated from the galaxy extractions.

3. The effective volumes for each field/subtype are found and multiplied by the space densities per subtype. The results for all the WISP fields are added, yielding a surface density for each subtype for the entire survey (Figure 2).

The expected surface density of each spectral type across the entire WISP survey is summarized in Figure 2 for different assumed power-law slopes in the low-mass stellar mass function. The number of dwarfs found is roughly consistent with expectations; in particular, the fact that we find no early-type $(<\mathrm{T} 4)$ dwarfs, despite their higher intrinsic luminosities, is consistent with their rarity (Burgasser 2007). The total of three $\mathrm{T}$ dwarfs found is consistent with other field mass function estimates based on wide, shallow surveys of T dwarfs (e.g., Metchev et al. 2008; Burningham et al. 2010). However, finding

\footnotetext{
10 We require $\mathrm{S} / \mathrm{N}=10$ in this analysis, an approximation based on simple tests with the WFC3 spectra. However, changing the $\mathrm{S} / \mathrm{N}$ requirement can significantly influence the expected surface density of dwarfs.
} 

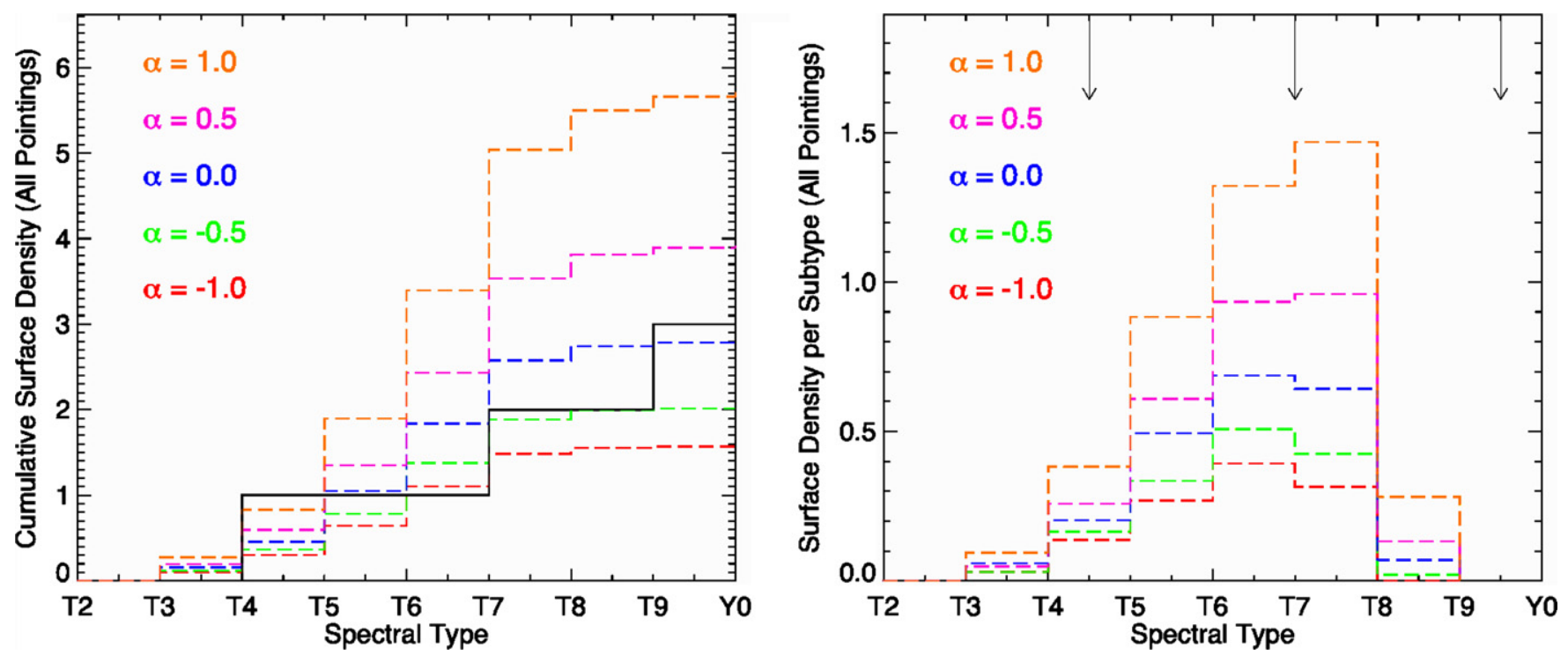

Figure 2. Left: expected cumulative surface density of late-type dwarfs across all WISP fields for different values of $\alpha$ in the power-law stellar mass function $d N / d M \sim M^{-\alpha}$, from the simulations described in Section 6. The total number found (three, black line) is generally consistent with $\alpha=0.0-0.5$. Right: the expected surface density of each spectral type across the entire WISP survey. The spectral types of the three dwarfs found are indicated with downward arrows. The discovery of a T9.5/Y0 dwarf is somewhat surprising; see Section 6 for a discussion of possible explanations.

a T9.5/Y0 dwarf is somewhat surprising due to their faintness. Possible explanations for this include (1) a flatter absolute magnitude/spectral-type relation than preliminary estimates indicate (Cushing et al. 2011; Kirkpatrick et al. 2011); (2) a sudden upturn in the space density of very-late-type dwarfs, possibly associated with a nearby free-floating planetary population (Sumi et al. 2011) or a larger proportion of old halo brown dwarfs at higher scale heights (for this simulation, a 1:400 halo fraction was assumed based on Digby et al. 2003); (3) a fortuitous discovery; or (4) some combination of the above. We note that WISP $1305-2538$ is 4 '.2 from the very-low-mass triple Kelu $1 \mathrm{ABC}$ at $18.7 \pm 0.7 \mathrm{pc}$ (Dahn et al. 2002; Liu \& Leggett 2005; Stumpf et al. 2008). With a 5000 AU projected separation, this matching is unlikely (Dhital et al. 2010), but second-epoch imaging would allow us to affirm or refute the existence of a widely separated, very low mass quadruple. Additionally, follow-up proper-motion measurements of all three new dwarfs would help establish whether they are galactic halo members.

\section{SUMMARY}

We have presented three distant $\mathrm{T} / \mathrm{Y}$ dwarfs spectroscopically confirmed by the WISP survey. These discoveries show the power of $H S T$ /WFC3 slitless grism spectroscopy to simultaneously find these objects at much larger distances than has been possible with ground-based surveys and yield accurate spectral types, making surveys such as WISP powerful new probes of the population of cold brown dwarfs in the galaxy.

We thank an anonymous referee for helpful comments that improved this Letter. This research has benefitted from the SpeX Prism Spectral Libraries, maintained by Adam Burgasser at http://www.browndwarfs.org/spexprism. We also thank Michael Cushing for providing Y0 spectra from the WISE survey. This work has been supported by the Carnegie Observatories Graduate Research Fellowship.

\section{REFERENCES}

Atek, H., Malkan, M., McCarthy, P., et al. 2010, ApJ, 723, 104 Brammer, G., van Dokkum, P., Franx, M., et al. 2012, arXiv:1204.2529 Burgasser, A. J. 2004, ApJS, 155, 191

Burgasser, A. J. 2007, ApJ, 659, 655

Burgasser, A. J., Cruz, K. L., Cushing, M., et al. 2010, ApJ, 710, 1142

Burgasser, A. J., Geballe, T. R., Leggett, S. K., Kirkpatrick, J. D., \& Golimowski, D. A. 2006, ApJ, 637, 1067

Burgasser, A. J., Kirkpatrick, J. D., Brown, M. E., et al. 2002, ApJ, 564, 421

Burgasser, A. J., Kirkpatrick, J. D., Burrows, A., et al. 2003, ApJ, 592, 1186

Burningham, B., Pinfield, D. J., Lucas, P. W., et al. 2010, MNRAS, 406, 1885

Burrows, A., Marley, M., Hubbard, W. B., et al. 1997, ApJ, 491, 856

Chabrier, G. 2001, ApJ, 554, 1274

Chiu, K., Fan, X., Leggett, S. K., et al. 2006, AJ, 131, 2722

Chiu, K., Liu, M. C., Jiang, L., et al. 2008, MNRAS, 385, L53

Cushing, M. C., Kirkpatrick, J. D., Gelino, C. R., et al. 2011, ApJ, 743, 50

Dahn, C. C., Harris, H. C., Vrba, F. J., et al. 2002, AJ, 124, 1170

Delorme, P., Willott, C. J., Forveille, T., et al. 2008, A\&A, 484, 469

Dhital, S., West, A. A., Stassun, K. G., \& Bochanski, J. J. 2010, AJ, 139, 2566

Digby, A. P., Hambly, N. C., Cooke, J. A., Reid, I. N., \& Cannon, R. D. 2003, MNRAS, 344, 583

Dupuy, T. J., \& Liu, M. C. 2012, arXiv:1201.2465

Geballe, T. R., Knapp, G. R., Leggett, S. K., et al. 2002, ApJ, 564, 466

Kirkpatrick, J. D., Cushing, M. C., Gelino, C. R., et al. 2011, ApJS, 197, 19

Kirkpatrick, J. D., Looper, D. L., Burgasser, A. J., et al. 2010, ApJS, 190, 100

Kirkpatrick, J. D., Reid, I. N., Liebert, J., et al. 1999, ApJ, 519, 802

Liu, M. C., Delorme, P., Dupuy, T. J., et al. 2011, ApJ, 740, 108

Liu, M. C., Dupuy, T. J., \& Leggett, S. K. 2010, ApJ, 722, 311

Liu, M. C., \& Leggett, S. K. 2005, ApJ, 634, 616

Lodieu, N., Pinfield, D. J., Leggett, S. K., et al. 2007, MNRAS, 379, 1423

Lucas, P. W., Tinney, C. G., Burningham, B., et al. 2010, MNRAS, 408, L56

Metchev, S. A., Kirkpatrick, J. D., Berriman, G. B., \& Looper, D. 2008, ApJ, 676, 1281

Nakajima, T., Oppenheimer, B. R., Kulkarni, S. R., et al. 1995, Nature, 378, 463 Oppenheimer, B. R., Kulkarni, S. R., Matthews, K., \& Nakajima, T. 1995, Science, 270, 1478

Pirzkal, N., Burgasser, A. J., Malhotra, S., et al. 2009, ApJ, 695, 1591

Reid, I. N., Kirkpatrick, J. D., Liebert, J., et al. 1999, ApJ, 521, 613

Ryan, R. E., Thorman, P. A., Yan, H., et al. 2011, ApJ, 739, 83

Scholz, R.-D., Bihain, G., Schnurr, O., \& Storm, J. 2011, A\&A, 532, L5

Strauss, M. A., Fan, X., Gunn, J. E., et al. 1999, ApJ, 522, L61

Stumpf, M. B., Brandner, W., Henning, T., et al. 2008, arXiv:0811.0556

Sumi, T., Kamiya, K., Bennett, D. P., et al. 2011, Nature, 473, 349

Wright, E. L., Eisenhardt, P. R. M., Mainzer, A. K., et al. 2010, AJ, 140, 1868 